\title{
Coulisses
}

Revue de théâtre

14 | Printemps 1996

Varia

\section{Association Internationale du théâtre à l'Université}

\section{(2) OpenEdition}

1 Journals

Édition électronique

URL : http://journals.openedition.org/coulisses/4775

DOI : $10.4000 /$ coulisses. 4775

ISSN : 2546-9460

Éditeur

Presses universitaires de Franche-Comté

\section{Édition imprimée}

Date de publication : 1 mai 1996

Pagination : 64-68

ISSN : 1150-594X

\section{Référence électronique}

"Association Internationale du théâtre à l'Université », Coulisses [En ligne], 14 | Printemps 1996, mis en ligne le 20 mars 2019, consulté le 24 septembre 2020. URL : http://journals.openedition.org/ coulisses/4775; DOI : https://doi.org/10.4000/coulisses.4775

Ce document a été généré automatiquement le 24 septembre 2020.

Coulisses 


\section{Association Internationale $\mathrm{du}$ théâtre à l'Université}

Coulisses $\mathrm{n}^{\circ} 11$, janvier 1995 , avait consacré un dossier à la situation du théâtre universitaire dans le monde à l'occasion de la création de l'Association Internationale des Théâtres Universitaires. Ce dossier comprenait la charte de cette association ainsi que des articles destinés à rendre compte de l'esprit des différentes tables rondes. Depuis, le Comité exécutif s'est réuni dans le cadre de la Biennale du Théâtre à l'Université de Murcie et Alicante le 9 novembre 1995, puis à Liège lors des $13^{\mathrm{e}} \mathrm{RITU}$ en février 1996. II nous semble utile de publier des extraits des rapports de synthèse des différentes tables rondes du premier congrès et de mettre en évidence l'évolution de l'Association en donnant les points forts des deux autres réunions.

\section{Premier congrès mondial du Théâtre à l'Université}

\section{3-15 octobre 1994, Liège}

Rapport de synthèse des différentes tables rondes

Extraits

\section{Table ronde $\mathrm{n}^{\circ} \mathrm{I}$ : La diversité des expériences du TU}

Rapporteur : Philippe Rouyer, Université de Bordeaux III

1 Étaient présents à la table ronde et ont proposé des communications: Les représentants des théâtres universitaires de Bologne, de Tel Aviv, de Rabat, d'Agadir, de Bulgarie, d'Espagne, de Penh, de Neuchâtel, d'Athènes, de Besançon, du Sri Lanka. M. Watanabé, professeur de littérature française à l'université de Tokyo, metteur en scène, représentant du ministère japonais de la culture, a fait une communication sur le théâtre au Japon (TU et théâtre en général).

2 1. A travers les communications et les discussions qui ont suivi, la diversité extraordinaire des pratiques et la variété des expériences à des niveaux divers (administratif, organisationnel, types de diplômes, recrutement de quel type d'enseignants avec quels diplômes - une mise en scène n'est-elle pas pour un professionnel l'équivalent d'une thèse ? - pédagogique, social, politique et esthétique) 
un besoin s'est manifesté : chacun d'entre nous devrait se mettre en devoir d'écrire une histoire du théâtre universitaire dans son pays.

3 2. Malgré cette diversité une définition générale du théâtre universitaire est cependant apparue : le théâtre en université est un outil éducatif au sens large et on peut dégager une typologie utile. Le théâtre est éducatif au niveau personnel et académique (au sens anglais de "académie ») ; le théâtre en université est un outil de pédagogie et de citoyenneté, un outil de développement culturel car aujourd'hui l'origine socioculturelle des étudiants change ; c'est aussi un outil de représentation devant un public, mieux des publics. La désignation théâtre universitaire comporte des connotations un peu magiques.

4 2.1. Le théâtre est un outil de développement personnel au sein d'une communauté où on suit des études universitaires (qu'elles soient liées aux arts et lettres, aux arts du spectacle ou à toute autre forme de cursus (scientifique, juridique, économique). Le théâtre en université ( $\mathrm{TU} »)$ est un instrument à la fois social et artistique propre à développer des relations communautaires et ludiques: on se retrouve pour prendre conscience de sa personnalité et trouver du plaisir partagé.

2.2. Le TU peut être un moyen d'apprentissage d'une langue étrangère in situ et de la culture qu'elle véhicule.

6 2.3. Le TU est un baromètre des problèmes qui se posent au théâtre dans chaque pays et de l'état de sa société sur tous les plans (moral, social, politique et esthétique).

7 2.4. Le TU peut être un instrument de prise de conscience d'une identité nationale par la langue et la culture qu'elle porte, dans un État qui ne reconnaît pas cette identité nationale; affirmer une nationalité sans État est le but du théâtre en université en Galice.

8 2.5. Le TU doit permettre de retrouver des racines profondes et des traditions ancestrales cachées par notre société trop tournée vers les média et le spectacle commercial ; les modalités sont, bien entendu, diverses selon les pays (Maroc, Japon...).

2.6. Le TU est un lieu d'expérimentation (d'avant-garde ?) et d'expérience de la liberté créatrice qui n'est pas seulement esthétique (rapports avec le théâtre officiel et professionnel) mais aussi sociale et politique ; le théâtre universitaire est à côté, contre, en marge, il se démarque du théâtre en général; le théâtre universitaire doit être reconnu mais pas rendu officiel car il pourrait y perdre sa liberté, même si on lui donnait des moyens (budget, salle, etc.). Le TU doit être un creuset pour la découverte de nouvelles formes de discours théâtral par la pratique; il se doit d'inventer un métalangage (S. Tampalini), piste qu'il faudra creuser.

10 3. Le théâtre en université est-il un théâtre amateur ou non ? Ni oui ni non.

11 3.1. Dans ses rapports avec l'université : doit-il être reconnu, officiel, accepté, autorisé, autonome, marginal ? Avec toutes les conséquences que cela entraîne au niveau des moyens financiers et matériels. Ces différentes questions sont plus importantes que de savoir si le TU est amateur ou non.

Quelle doit être la place du théâtre dans les études universitaires? Spécialisé avec cursus complet en études et pratique théâtrale, en études théâtrales seulement ou en pratique théâtrale uniquement? Tous les étudiants peuvent-ils faire du théâtre quel que soit leur cursus et faire valider dans leur cursus ces études théâtrales même si ce sont des étudiants en droit ou en sciences?

Quel personnel doit enseigner dans les études et la pratique théâtrale? Un personnel 
formé académiquement et/ou professionnellement ? Quels diplômes l'université exiget-elle pour les recruter?

Quel est le but du TU: former des professionnels dans tous les secteurs du théâtre (acteur, metteur en scène, technicien) ou des généralistes qui deviendront à leur tour des formateurs? Ou former des citoyens épanouis par l'expérience de la liberté sur la scène?

3.2. Y-a-t-il une différence de nature ou de degré entre TU et théâtre professionnel ? Sur le plan esthétique il n'est pas nécessaire de pratiquer systématiquement la comparaison entre TU et théâtre professionnel, théâtre amateur et non amateur ; on ne fait pas exactement la même chose du théâtre ou avec le théâtre. Le TU peut apporter une contribution érudite fondamentale à des troupes professionnelles (cas du Groupe Antique de Neuchâtel).

La bonne solution consiste sans doute à faire que des professionnels (auteurs, metteurs en scène, décorateurs, éclairagistes, etc.) participent à la réalisation de spectacles de TU.

13 3.3. Le répertoire du $\mathrm{TU}$ est une vraie question; à travers le choix du répertoire se dessine une pratique sociale, politique par rapport à la société. Faut-il rejeter le répertoire classique, le subvenir, écrire du neuf collectivement ou individuellement? Le TU est peu préoccupé de rentabilité à la différence du théâtre professionnel où la rentabilité tend à devenir le seul critère de qualité. Le TU peut aider le théâtre à ne pas perdre son âme et à garder sa capacité de dire des choses neuves dans des formes neuves sur de nouvelles pratiques sociales.

14 4. Tout ceci a conduit la table ronde à se poser la question très importante du public qui assiste aux représentations de $\mathrm{TU}$ : à qui s'adresse-t-il ?

Le problème de la qualité mais aussi des modèles culturels est implicite dans cette approche.

15 A travers l'expérience de Cymbeline menée par L.Garbagnati avec cinq pays et 80 participants, on se rend compte que le TU forme au théâtre par le théâtre ; les acteurs d'aujourd'hui sont le public de demain ; mais par le jeu des amitiés, les copains de ceux qui font du théâtre prennent peu à peu conscience que le théâtre, c'est aussi pour eux ; tous ensemble ils construisent un public.

16 Mais il ne faut pas que le TU vive en autarcie; il doit se produire hors des murs de l'institution chaque fois qu'il le peut et il doit le vouloir ; à cet égard les festivals de TU doivent être ouverts à un large public non étudiant. Parmi les étudiants il faut cependant noter que beaucoup d'entre eux ne vont pas au théâtre car les modèles que leur offre le théâtre, disons commercial pour faire vite, ne leur plaisent pas; de plus ils pensent qu'en général TU est synonyme de théâtre intellectuel et difficile. On peut même parler de boycott du TU par les étudiants ; il s'agit donc de bien réfléchir au type de répertoire à présenter. Faire du TU, c'est ne pas oublier qu'on joue aussi pour un public et pas seulement pour se faire plaisir devant des salles vides.

17 Dans les festivals, il s'agit de retrouver le sens de la fête communautaire, une certaine spontanéité du produit non encore fini; il ne s'agit pas de vendre; il faut savoir conserver un certain amateurisme à une époque où tout le monde veut passer pour professionnel ; pouvoir improviser et modifier à la dernière minute fait partie du jeu théâtral dans le TU. Certes, il faut poser le problème de la qualité ; on reprendra ici la belle image de la grappe de raisin de notre collègue hongrois : un TU c'est une grappe : 
quels grains de la grappe sont les meilleurs? Lesquels va-t-on faire goûter à quels spectateurs, dans quels festivals?

Le TU est à la croisée des chemins; il est la caisse de résonance de tous les problèmes socio-culturels qui traversent le milieu étudiant et la société en général; dans un paysage où les professionnels et les universitaires entretiennent encore (toujours?) trop souvent des relations de méfiance souvent économique (le TU qui a du succès est un concurrent potentiel, voire réel) mais aussi culturelle; les universitaires qui encadrent couramment les groupes de TU pensent qu'ils connaissent le théâtre; les professionnels pensent trop souvent que les universitaires (qu'ils confondent avec le $\mathrm{TU})$ défendent un théâtre soit trop expérimental soit trop traditionnel. Or la chance du TU dans ses relations avec le théâtre en général (et vice versa) est de pouvoir proposer des pistes de renouvellement, de formuler des propositions neuves sans pour autant vouloir imposer un répertoire académique et traditionnel car le théâtre professionnel de son côté est devenu un peu trop préoccupé de rentabilité.

\section{Table ronde $\mathrm{n}^{\circ} 2$ : Création, formation, recherche}

Rapporteur : Jean-Marc Larrue, collège de Valleyfield, Québec.

Cette table ronde a été à l'image de notre jeune association ; elle a montré la diversité des pratiques théâtrales et des intérêts qui s'investissent dans le TU; elle a prouvé nous avions besoin de nous écouter pour nous connaître et tirer parti de nos diversités. 20 intervenants pour 18 institutions de 14 pays, c'est un beau panorama. La synthèse sera rapide mais précise sur les trois points de la table ronde.

L'activité théâtrale de formation est importante; on s'aperçoit que le théâtre universitaire peut être un moyen d'éducation populaire auprès des habitants des villages qui entourent une université. On se rend compte qu'en France les grandes écoles d'ingénieurs prestigieuses encouragent la pratique théâtrale pour faire des futurs cadres de vrais citoyens capables de relations humaines.

Au Québec, le théâtre a pu être obligatoire comme moyen de développement cognitif et personnel; au Mexique une expérience scientifique est en cours qui vise à mesurer les effets de la pratique théâtrale sur les changements cognitifs des jeunes acteurs universitaires ; aux Pays-Bas, le théâtre est une matière officielle dans les programmes du secondaire ; il ne s'agit pas tant de former des professeurs de théâtre que de donner aux futurs enseignants une méthodologie pédagogique qui valorisera leur formation académique. En Argentine des expériences sont en cours pour évaluer la capacité de l'activité théâtrale à développer la capacité cognitive et mentale de ceux qui pratiquent le théâtre. En Grande-Bretagne ("Chasing Shadows»), au Danemark, des expériences pointues sur l'apport culturel du théâtre sont en cours.

21 Le TU peut aider à la mise au point d'analyses d'œuvres qui seront utilisables ensuite par des professionnels dans leurs productions. Le TU peut établir des ponts entre recherche, théorie et pratique.

Quel est le statut du théâtre en université ? S'agit-il de faire de la pratique théâtrale ? de donner une formation universitaire professionnelle (Glasgow, Tel Aviv, Buenos Aires) ? de former des professionnels sur le plan théorique, donc de faire des études surtout théoriques? Il faut que le TU reste une pratique pour conserver dynamisme et vie. 


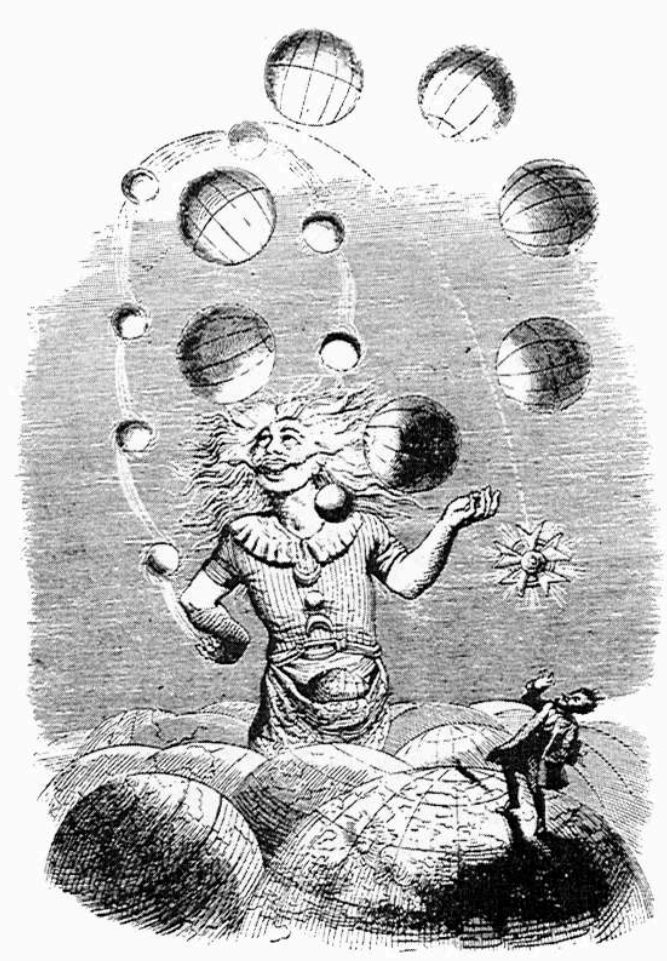

A l'issue des communications, les discussions ont été vives et fructueuses; elles peuvent se résumer autour de quatre points.

1. Il est essentiel de définir ce que sont nos concepts clés en théorie comme en pratique et voir quels sont les rapports dans les universités entre recherche théâtrale et pratique, entre recherche et enseignement, entre pratique et enseignement.

2. Quelle place occupe le théâtre dans l'université par rapport à la formation en général ? L'université est-elle au service du théâtre comme art ou comme industrie? Peut-on se contenter dans l'université de recherches théoriques?

3. La spécificité de l'université et du TU, c'est de pouvoir faire à la fois de la recherche et de la pratique, ce que peu d'autres institutions peuvent faire en même temps : l'approche multidisciplinaire est ce qui peut faire avancer l'université : créer des ponts entre les disciplines (sociologie, psychologie, etc.).

4. Il est temps de définir une épistémologie propre à la recherche et à la pratique en milieu universitaire. Il faut imaginer, constituer des modèles de recherche et de pratique, de création applicables à cette pratique et qui en découlent; il est normal qu'en milieu universitaire on songe à ouvrir des voies (cf. en Grande-Bretagne « Chasing Shadows » ou en Argentine).

Il revient à notre association de mettre au point une prospective des besoins à venir : il faut sans doute viser à former des acteurs-chercheurs plus que des acteurs ou des chercheurs.

En conclusion, la table ronde propose des balises pour le prochain congrès: des modalités et un principe :

- sur des expériences et sur les intérêts bien exposés de ces expériences ;

- sur les écoles professionnelles en université ;

- sur les études théoriques ; 
- sur les troupes de TU.

évident pour tous ; mais que faire après qu'on ait appris à se connaître ? Les différences entre le cursus théâtre dans les universités sont considérables dans le monde. Dans l'esprit qui est celui de l'ONU, il existe des possibilités d'échange entre professeurs et étudiants pour partager leurs compétences et pratiquer une entraide active. Ce type d'action est à développer au sein de l'AITU.

2. La mise en place des commissions nationales (au sens d'aires linguistiques) permettrait de poursuivre des objectifs nationaux communs comme l'organisation de festivals, des procédures d'assistance mutuelle, de définition d'une philosophie commune, etc. Ces groupes se réuniraient lors des congrès AITU. La solidarité est une valeur forte.

31 3. Le groupe s'est posé la question du public du théâtre universitaire mais personne ne s'est vraiment demandé ce qu'était un étudiant en théâtre : voilà une piste à suivre. Les participants à cette table ronde ont été unanimes à dire que le théâtre comme source de connaissance et d'expérience devait être mis à la portée des étudiants à une époque tournée vers l'électronique et dont la fascination pour l'ordinateur fait de cet outil un instrument privilégié de recherche et d'expression. La structure profonde de notre vie aujourd'hui est façonnée par les média; leurs audaces, leur complexité envahissent 
notre vie de tous les jours. Sous cet éclairage, quelle peut être la nature du théâtre dans le monde d'aujourd'hui?

4. Plusieurs participants ont évoqué le besoin de comprendre la relation qui s'établit entre le travail sur les matériaux bruts de la création théâtrale et leur mise en forme artistique. La fonction du théâtre dans le monde universitaire a été décrite comme le besoin de donner aux études théâtrales à l'université le statut d'une discipline à part entière (théorie et pratique) afin de :

- présenter des conclusions sous une forme attrayante et dans un souci d'action positive,

- explorer les vastes champs de la connaissance et les capacités de l'intelligence humaine,

- approfondir l'expérience humaine pour développer notre compréhension des conditions sociales existant aujourd'hui,

- révéler ce qu'il y a de meilleur dans l'humanité.

\section{Réunion du Comité exécutif}

Alicante, 9-10 novembre 1995

Étaient présents des représentants des TU d'Allemagne, d'Argentine, d'Australie, de Belgique, du Canada, d'Ecosse, d'Espagne, d'Israël, du Mexique, de Pologne. organismes pertinents (université, festivals) et de personnes impliquées dans des activités théâtrales universitaires. Il leur apparaît primordial d'insister dans ce processus sur l'apport concret de l'AITU qui pourrait être en conformité avec la charte :

- de favoriser les échanges entre les festivals et de les faire connaître ;

- d'aider à la recherche et à l'obtention de subventions ;

- de favoriser l'échange d'informations relatives au théâtre à l'Université (par exemple, par un bulletin régulier aux membres).

Un congrès mondial a également été préparé pour le printemps 1997. Le document préparatoire a été déposé par Jean-Marc Larrue et préparé conjointement avec Luis Thenon. Ce congrès aura lieu successivement au Collège de Valleyfield (au Québec) et à l'université Laval (à Québec). Une date restant à confirmer a été proposée : du 8 au 12 mars 1997, c'est-à-dire la première période disponible car elle coïncide avec la relâche semestrielle à l'Université Laval et au Collège de Valleyfield.

Les participants ont terminé leur réunion en considérant que l'AITU - IUTA se présente avant tout comme un moyen de communiquer pour les théatres à l'université, un réseau pour que se retrouvent des gens qui ont des intérêts communs.

\section{Colloque AITU dans le cadre des $13^{e}$ RITU de Liège}

\section{Vendredi $1^{\text {er }}$ mars 1996}

Ce colloque a réuni de nouveaux représentants dont ceux des TU d'Allemagne, d'Australie, d'Autriche, de Belgique, de Croatie, de France (Reims, Lyon), d'Israël, d'Italie, du Kansas, du Maroc, de Pologne, de Tunisie et d'Ukraine.

La commission des moyens :

Il convient d'organiser un groupe moteur ou un secrétariat permanent qui puisse 
proposer des projets établis sur dossier afin d'obtenir les financements nécessaires, financements qui suivent généralement. Assurer le suivi est d'ailleurs essentiel. On peut penser à ce sujet que les dossiers envoyés à l'I.I.T., au

Programme de Participation de l'UNESCO, aux Communautés Européennes (Kaléidoscope) n'ont pas abouti faute de suivi et de réponses rapides.

41 Cette commission pourra ainsi centraliser les informations venues des différents membres qui pourraient donner les moyens, les «trucs» ou «ficelles» (jumelage, alliance, sponsors...) que chacun développe chez lui pour l'obtention de subventions. L'AITU pourrait ainsi informer mais aussi passer au stade de «conseil» et d'aide concrète.

Il ne faut pas oublier que l'AITU étant une association internationale a automatiquement droit - et ce n'est pas uniquement une possibilité - à des aides de niveau international. 